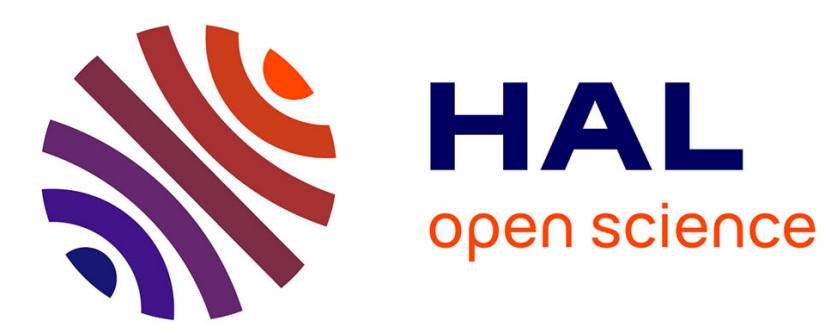

\title{
La réforme de la politique scientifique française face à la mondialisation: l'émergence incertaine d'un nouveau référentiel d'action publique
}

\author{
Anne Branciard, Eric Verdier
}

\section{- To cite this version:}

Anne Branciard, Eric Verdier. La réforme de la politique scientifique française face à la mondialisation : l'émergence incertaine d'un nouveau référentiel d'action publique. Politiques et Management public, 2003, 21 (2), pp.61-81. halshs-00153797

\section{HAL Id: halshs-00153797 \\ https://shs.hal.science/halshs-00153797}

Submitted on 13 Jun 2007

HAL is a multi-disciplinary open access archive for the deposit and dissemination of scientific research documents, whether they are published or not. The documents may come from teaching and research institutions in France or abroad, or from public or private research centers.
L'archive ouverte pluridisciplinaire HAL, est destinée au dépôt et à la diffusion de documents scientifiques de niveau recherche, publiés ou non, émanant des établissements d'enseignement et de recherche français ou étrangers, des laboratoires publics ou privés. 


\section{LA REFORME DE LA POLITIQUE SCIENTIFIQUE FRANÇAISE FACE A LA MONDIALISATION : L'EMERGENCE INCERTAINE D'UN NOUVEAU REFERENTIEL D'ACTION PUBLIQUE par}

Anne BRANCIARD et Eric VERDIER

Laboratoire d'économie et de sociologie du travail (LEST, UMR 6123)

\section{RESUME :}

L'enjeu principal de cette contribution tient dans sa tentative de rendre compte du travail de médiation qui articule des répertoires d'interprétation du monde la recherche et de la technologie à la formulation des politiques publiques et, en particulier, des réformes récentes. Autrement dit, il s'agit d'analyser le passage d'une posture analytique à des préconisations normatives. Dans cette perspective, les rapports d'experts tiennent une place cruciale.

Les politiques scientifiques et les réformes qu'elles ont connues en France constituent un domaine d'élection pour une "approche cognitive" de l'action publique. En effet, ainsi que ce texte s'attachera à le montrer, l'analyse de ces réformes et, en arrière-plan, de la trajectoire du dispositif français de recherche et de technologie, est inséparable de l'appropriation des analyses produites par la sociologie et l'économie de l'innovation et des connaissances. Elles sont médiatisées par des rapports d'expertise commandités par les pouvoirs publics nationaux et/ou élaborées par les organisations internationales, au premier rang d'entre elles, l'OCDE.

Comme dans d'autres domaines de l'action publique, l'élaboration des récentes réformes est souvent assimilée à une promotion des régulations marchandes. Certes, c'est bien la construction des années 50-70, qualifiée de 'colbertiste' ou de 'régulation administrée', qui est ainsi en jeu ; mais on peut avancer qu'une conception connexionniste de la science et de la technologie, mariant les réseaux et l'interaction tournées vers l'élaboration de projets, tend à prédominer. Dans les faits, c'est une pluralité de compromis et d'agencements entre différents principes de justification qui semble prévaloir. Il en résulte une forte indétermination des trajectoires sociétales. 
Ces dernières années, de nombreux rapports officiels ont été consacrés aux performances et aux réformes envisageables du système français d'enseignement supérieur et de recherche. Serait ainsi en cours la constitution d'un référentiel d'action publique (Jobert, Muller, 1987), visant à permettre à ce dispositif institutionnel de faire face aux enjeux de la compétitivité par la connaissance, dans le cadre de la globalisation économique et de l'européanisation des politiques publiques ${ }^{1}$. Sans conteste, le « rapport Guillaume »(1998), du nom de son auteur, ancien Commissaire au Plan puis directeur de l'Agence Nationale pour la Valorisation de la Recherche (ANVAR), a établi les fondements de ce référentiel. En effet, dans ses attendus, la loi sur l'innovation et la recherche de 1999, tout comme les rapports parlementaires qui l'ont précédée et suivie y font constamment référence. L'expertise scientifique, relayant les analyses savantes de la recherche et de l'innovation, a ainsi très rapidement trouvé une traduction politique, non seulement dans de nouveaux principes d'action mais aussi dans une série de mesures concrètes, programmes et incitations pour les entreprises et les acteurs du champ de la recherche.

La première partie de ce papier rappelle les diagnostics et critiques des discours d'experts nourris des interprétations des travaux de recherche sur l'économie et la sociologie de la science et de l'innovation, à partir desquels certains acteurs cherchent à légitimer le changement. La seconde s'attache à rendre compte de l'émergence de nouvelles orientations qui, avec le soutien de l'expertise internationale institutionnalisée, tente de s'ériger en nouveau référentiel d'action publique (Branciard, 1999).

\section{LES DIAGNOSTICS FONDATEURS : L'INADAPTATION CROISSANTE DE L'INTERVENTION PUBLIQUE A LA NOUVELLE DONNE DE LA RECHERCHE ET DE L'INNOVATION}

\section{L'affirmation d'un cadre interprétatif : le Mode 2 de production des savoirs}

Durant les années 90 - mais les origines sont bien antérieures -, s'affirment des schémas d'analyse qui s'écartent du classique modèle séquentiel linéaire allant de la recherche

\footnotetext{
${ }^{1}$ Voir à ce sujet Attali (1998) et van Dyck (2001).
} 
fondamentale à la technologie puis au développement de produits. Ils mettent en avant l'articulation des interdépendances et interactions entre les différents segments de l'ensemble du système gouvernant la production, la diffusion et l'utilisation du savoir économiquement valorisable. Ils vont partiellement dans le sens d'une description d'une extension des pratiques de la science connectées à des espaces productifs à valorisation économique et /ou sociale, et une interpénétration croissante entre la recherche de «connaissances objectives » (i.e. la production de savoirs académiques présentés comme «socialement neutres ») et la recherche d'efficacité. L'émergence d'un «nouveau » régime de production des savoirs a été formalisé par des auteurs anglo-saxons dans un ouvrage édité par Gibbons, «The new production of knowledge, the dynamics of science and research in contemporary societies »(1994). Ce «mode 2 » serait centré sur les problèmes à résoudre tels qu'ils sont définis par l'industrie, en rupture avec un «mode $1 »$ plus traditionnel et disciplinaire dans lequel les problèmes étaient posés et résolus dans un contexte gouverné par les intérêts d'une communauté spécifique largement académique.

Cette thèse d'une supplantation d'un régime de production de la science par un autre est critiquée par D. Pestre (1997), pour lequel les deux modes sont parallèlement à l'œuvre depuis plusieurs siècles en Occident. La formalisation du Mode 2 de Gibbons et alii renverrait donc davantage à un changement de norme culturelle et de «croyances », qu'à la validité empirique d'un changement de pratiques scientifiques. Elle tend à légitimer un rapprochement et, plus encore, des interactions organisées entre le monde académique et l'entreprise (Shinn, 2002) (in Branciard, op.cit.).

\section{Le constat initial: Une recherche scientifique honorable et des performances technologiques insuffisantes}

Le constat de base du rapport Guillaume tient à l'écart entre des positions scientifiques jugées plutôt correctes et qui, sur moyenne période, sont en progrès, et des résultats technologiques, notamment en terme de brevets déposés, assez médiocres. Ce constat est repris dans tous les discours officiels, notamment durant les débats préparatoires à la loi du 12 juillet 1999 sur l'innovation et la recherche: «L'innovation et la recherche constituent en France un curieux binôme. En effet, notre recherche obtient d'excellents résultats alors que la capacité de nos entreprises à innover reste relativement décevante » (Chevallier, 1999).

De cet étalonnage général des performances nationales, découle très explicitement le «diagnostic (...) sans appel [du rapport Guillaume]: «La France n’utilise pas avec 
l'efficacité souhaitable son potentiel de recherche pour dynamiser l'ensemble de son tissu économique et pour créer de nouvelles entreprises dans des secteurs où sa spécialisation est la plus faible»(Chevallier, ibid., ). Ce diagnostic est issu essentiellement des travaux de l'Observatoire de la Science et de la Technologie, créé au début des années soixante-dix et présidé par un ancien directeur général du CNRS (Pierre Papon). Ces indicateurs de l'OST, (2000) assurent une médiation essentielle entre les analyses issues de la recherche et des diagnostics politiques. L'analyse de cette défaillance des performances technologiques se réfèrerait à trois causes principales :

Une sous-exploitation des potentialités ouvertes par l'avènement d'une "société de la connaissance »

La notion de «knowledge-based economy » est fondée sur la rencontre d'une tendance lourde et d'un événement technologique majeur (Foray, 2000b, ) :

- les procès de production sont de plus en plus basés sur des activités incorporant des savoirs comme facteur de production et, en outre, les secteurs à haut degré de connaissances scientifiques et technologiques tendent à supplanter, comme moteur du développement économique, les secteurs traditionnels à procès plus stables et appuyés sur une forte proportion de main d'œuvre d'exécution.

- le développement accéléré des technologies de l'information et de la communication amplifie considérablement les processus de codification de la connaissance et de sa réduction en «information», ce qui facilite son transfert, son stockage et sa reproduction à moindre coût.

Ainsi la connaissance deviendrait également accessible à tous dans un monde entièrement «globalisé »; encore faut-il être capable de valoriser ce bien économique, susceptible d'engendrer des externalités positives considérables, comme l'avait montré K. Arrow dès les années soixante, afin d'espérer être un acteur à part entière de cette «knowledge society ». Dès le début des années 90, le Livre Blanc de la Commission des Communautés Européennes sur la «société de la connaissance » (1993) fait de cette dernière une ressource essentielle pour la croissance, la compétitivité et l'emploi des pays de l'Union européenne.

Les secteurs de haute technologie, fondés sur les sciences, en premier lieu les NTICs et les biotechnologies, sont considérés comme les moteurs privilégiés d'un développement durable. La maîtrise de leur développement constitue donc un fort enjeu national de compétitivité internationale. A cet égard, le retard accumulé par la France dans ces domaines stratégiques 
augurerait mal de son positionnement de moyen terme comme l'ont pointé en 1999 le rapport de deux parlementaires, reprenant en cela des constats du rapport Guillaume et de l'OST : «La position relative de la France est très médiocre, moins au niveau de son potentiel de recherche que de ses capacités technologiques et industrielles, notamment : l'ensemble des biotechnologies, l'ensemble des NTIC (Cohen, Le Déaut, 1999, )Plus généralement, en cohérence avec une conception informationnelle de la connaissance, la cause majeure de l'insuffisante «valorisation de la science » est un constat d'échec sur les dispositions de la loi de 1982 sur la recherche et de la loi de 1984 sur l'enseignement supérieur: «elles (...) ne débloquaient pas suffisamment de 'verrous' pour que les résultats de la recherche publique se traduisent par des créations d'entreprises et par l'accroissement des transferts à l'économie » (C. Allègre, Ministre de l'éducation nationale, de la recherche et de la technologie)

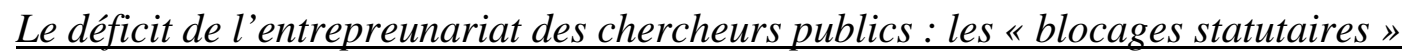

La dimension première a trait aux créations d'entreprises de pointe par les chercheurs publics, en vue de faire fructifier technologiquement les connaissances qu'ils ont élaborées. Or durant les années quatre-vingt-dix, il se créait tout au plus en France une trentaine de start up issues de l'essaimage de chercheurs, «ce qui, [aux yeux] des chercheurs et des capital-risqueurs français unanimes, ne reflète pas la richesse du potentiel scientifique et technologique français » (Guillaume, ibid.). Depuis 1984, seules 389 entreprises ont été créées à partir de la recherche publique alors qu'il s'en crée 1300 par an dans les secteurs de haute technologie (. Bret, 1999); la recherche française est donc en retard, non seulement sur les Etats-Unis (l'industrie de biotechnologies représente 150000 emplois hautement qualifiés) mais aussi sur le Canada, la Grande-Bretagne, l'Allemagne où une «dynamique naissante » s'est faite jour (ibid.).

L'accumulation de ces chiffres constitue une image globale qui, ultérieurement, inspirera une nouvelle politique et justifiera des mesures réglementaires et programmatiques. C'est ainsi que le Ministre renchérit en récapitulant cette activité de la recherche publique de la manière suivante : «soit une entreprise créée par an pour 1000 chercheurs ou enseignants-chercheurs » (Allègre, Assemblée Nationale 3/6/99).

La cause de ces difficultés est renvoyée prioritairement sur l'inadéquation des règles censées favoriser l'entrepreunariat des chercheurs.

L'insuffisante mobilité des chercheurs publics vers le monde marchand comme support des relations avec les entreprises 
«La mobilité des chercheurs statutaires vers les entreprises est quasi-inexistante» (Guillaume, op.cit.). Sur 10 ans, la Cour des Comptes (1997) évalue à 0,2 \% des effectifs en moyenne annuelle la mobilité depuis les EPST vers l'industrie «de l'ordre de 30 à 40 personnes par an sur un total de plus de 25000 chercheurs » (ibid., ) $)^{2}$.

Pourtant, la loi de 1982 avait posé le principe d'une fonctionnarisation des personnels de la recherche publique dans la mesure où les nouveaux statuts devaient «favoriser la libre circulation des idées et, sans préjudice pour leur carrière, la mobilité des personnels entre les divers métiers de la recherche au sein du même organisme, entre les services publics de toute nature, les différents établissements d'enseignement supérieur, et entre ces services et établissements et les entreprises » (cité par Chevallier, 1999). Le rapport Cohen, Le Déaut sur les priorités de la recherche parle explicitement de «contrepartie » à la titularisation, sous la forme d'une diversification des missions et [le développement de] la mobilité, (...) le filet de la titularisation [pouvant] conduire le chercheur à prendre des risques et à être mobile ».

On voit que le schéma d'analyse reste fortement empreint d'une conception assez linéaire des relations Science-Industrie, puisque la première est un "gisement», en l'occurrence mal exploitée par et pour la seconde. L'affinement du diagnostic et plus encore, la conception des remèdes, se sont appuyés sur des interprétations plus en phase avec des développements de ces vingt dernières années de la sociologie et de l'économie de l'innovation qui insistent sur l'importance des interactions entre «la République des Sciences » (la distribution égalitaire de l'information produite par la science ou «open science») et «le Royaume de la Technologie » (la nécessaire protection de la propriété intellectuelle des créateurs).

\section{Les difficultés françaises à structurer des relations Science-Industrie cohérentes avec les nouvelles modalités de production des savoirs}

\section{L'instrumentation du mode 2 dans les rapports d'expertise et parlementaires pour démontrer}

\section{l'inefficacité de la politique française}

Comme le constate le Rapport Guillaume, la traduction politique des thèses de Gibbons s'est imposée internationalement comme un «modèle normatif », et à ce titre, comme un élément clé d'un référentiel d'action publique. Quelle qu'en soit la validité empirique, cette conception, opposée au Mode 1, «conduit à une approche par le marché qui oblige à cerner les besoins latents mal identifiés, accepter la prise de risque de se lancer dans la réponse à un

\footnotetext{
${ }^{2}$ Tout en étant limités (800 à 900 bon an mal an), les flux inverses, des entreprises vers la recherche publique, sont
} 
besoin du marché qui passe souvent par une solution technique et scientifique pluridisciplinaire et maîtriser les problèmes fondamentaux juridiques, notamment de propriété industrielle, mais aussi de gestion liés à toute activité économique » (Duranton et alii, Conseil national d'évaluation de la recherche, 1999). L'adaptation à cette nouvelle donne bute sur deux types de difficultés: les unes relatives aux liens entre le monde académique et les firmes ; les autres qui tiennent à la faiblesse du soutien apportée aux start up.

Certes le nombre de relations contractuelles entre le CNRS et les entreprises a plus que décuplé entre 1983 et 1996 ; durant la même période, le montant total des contrats conclus par des sociétés privés avec des institutions de recherche publiques a augmenté de 8 fois (Laredo, Mustar 2001a). Malgré cet indéniable développement, prédomine l'idée selon laquelle les grands organismes scientifiques publics, à la notable exception de l'INRIA (Lanciano, Nohara, 2001), collaborent et/ou coopèrent insuffisamment avec le monde marchand.

Si les entreprises ont eu tendance à externaliser une partie de leurs dépenses de R\&D, elles n'en ont fait bénéficier que secondairement les laboratoires publics français (8,5\% en 1995, dont seulement $0,5 \%$ pour le CNRS) par rapport à d'autres composantes de leur groupe d'appartenance $(27,5 \%)$ ou encore à des firmes extérieures au groupe $(39,4 \%)$ ( Guillaume,ibid.).

\section{Le capital-risque est resté embryonnaire}

La question des financements apportés aux «jeunes pousses » de haute technologie peut être considérée comme emblématique de la rencontre des deux paradigmes évoqués jusqu'alors pour forger un nouveau contexte mondial de la recherche : une économie où la connaissance est érigée en bien économique et une place déterminante laissée à la résolution des problèmes posés par les firmes et plus largement la société (Barré, 2001).

A cet égard, le rapport Guillaume souligne que l'ampleur des fonds levés par les sociétés de capital-risque (1,5 MdsF en 1996 contre 40 MdsF aux Etats-Unis) est restée trop limitée pour offrir les perspectives de financement indispensables à la création d'entreprises de haute technologie. Les investissements en capital-risque ont certes progressé de $40 \%$ en 1997 par rapport à 1996 mais cette croissance a été de près de 90 \% en Allemagne qui a ainsi rattrapé le niveau français, lequel restait quatre fois moins moindre que celui du Royaume-Uni.

Au-delà de cette dimension technique, le diagnostic se veut «sociétal » et remonte aux modalités de prise en charge du risque industriel dans la société française : «Le financement

sensiblement plus importants grâce aux boursiers (environ 60 \% de l'ensemble) et en particulier, les boursiers CIFRE. 
privé des jeunes entreprises innovantes en France a traditionnellement fait dépendre les décisions d'investissement plus du niveau et de la nature des garanties que des espérances de profit. En faisant peser l'essentiel du risque sur le créateur et l'Etat, ce système aboutissait tout à la fois à un découragement de l'esprit d'entreprise, une mauvaise sélection des projets, et un sevrage des capitaux à des stades cruciaux de la vie de l'entreprise (naissance et sauts de croissance) » (OCDE citée par Trégouet, 1999)

\section{La remise en cause de l'organisation de l'action publique «à la française » se décline en plusieurs points}

\section{La dévalorisation d'un modèle mission-oriented et de sa version française qualifiée de} 'colbertiste'

La distinction entre politiques "mission-oriented" et "diffusion-oriented" est due à Ergas (1987) pour qualifier des ensembles caractéristiques de formes institutionnelles et de pratiques dans le domaine des politiques scientifiques et technologiques. Une politique "missionoriented" correspond à "des projets radicalement innovants, qui sont eux-mêmes nécessaires à la poursuite d'objectifs d'intérêt national". La mission concerne des domaines technologiques qui ont une signification stratégique pour l'Etat. Les traits principaux en sont la centralisation $\mathrm{du}$ processus de prise de décision, la définition d'objectifs dans des programmes gouvernementaux, la concentration du nombre de firmes impliquées, et la fondation d'une agence publique particulière à haut pouvoir discrétionnaire. La relation science/innovation est alors construite sur un modèle "colbertiste" ( Mustar, Laredo, 2001b) : c'est un modèle d'innovation 'top-down' «adapté aux objets technologiques complexes utilisés pour les grandes infrastructures publiques »(Barré, Papon, 2000). Ce modèle français s'est avéré particulièrement adapté à la réalisation d'objets de haute technologie relevant de marchés d'Etat (aéronautique, spatial, nucléaire, télécommunications ...). En outre, l'exécution repose largement sur un petit nombre de grandes firmes, alors qualifiées de «champions nationaux », en relation étroite avec des agences de financement ad hoc et des institutions de recherche appliquée publiques (création de l'INRIA, de l'ONERA, du CNES ...).

Il s'oppose, quasiment point par point, au modèle de l'innovation «par foisonnement »sélection par le marché ('bottom-up'), adapté aux opportunités d'hybridations sans fin des technologies actuelles, mais aussi à la tendance générale à la déréglementation des marchés à l'échelle internationale et, plus généralement, à la 'globalisation' »(Barré, Papon, ibid.). Il 
apparaît donc largement inadapté aux nouvelles normes de compétitivité associée à une « knowledge based society » ou encore aux «nouvelles » modalités de production des savoirs. Une politique "diffusion-oriented" se caractérise par son caractère décentralisé. Le rôle des agences publiques est restreint, l'accent est mis sur la conjonction de formes de recherche coopératives et d'institutions favorisant la diffusion systématique de la connaissance scientifique et technique. Les innovations sont censées émerger localement à partir d'initiatives de chercheurs-entrepreneurs soutenus par une multiplicité de partenariats. (in Branciard, 2001).

\section{Le grand programme technologique : une figure critiquée mais au déclin résistible}

Toute une série de critiques relatives à la régulation est associée au mode d'organisation par grands programmes, d'autant plus que, malgré une inadaptation aux nouveaux enjeux dénoncée tout au long de la décennie 90, il a paru ardu de s'en défaire. Ainsi la répartition de la dépense publique de recherche par discipline et programme reflèterait une spécialisation excessive attachée aux domaines de prédilection de cette politique de «mission » : en 1996 l'ensemble de l'aéronautique-aérospace, le nucléaire et l'autre R\&D militaire représentent plus de $37 \%$ des crédits. Les sciences de l'ingénieur ne drainent que 8,1\% des fonds publics (OST 2000).

Mais les critiques les plus vives des rapports cités se concentrent sur l'allocation des crédits aux entreprises provoquée par ce mode de coordination. En premier lieu, les financements budgétaires sont excessivement polarisés sur les grands groupes, au détriment des PME qui sont pourtant les plus dynamiques en matière d'innovation et de création d'emplois. Les trois premiers groupes bénéficiaires recevaient, en 1997, 42 \% des crédits incitatifs du ministère de l'économie, des finances et de l'industrie. En outre, pour ce qui est des crédits du programme cadre de recherche-développement de Bruxelles, on trouve une prédominance des «groupes qui bénéficient [déjà] de la concentration des crédits publics en France » (Guillaume, op.cit.). Enfin, ces groupes, qui bénéficient massivement des crédits publics, n'en diffusent guère les effets, puisqu'ils les consacrent pour la majeure partie à leur R\&D interne et recourent donc peu à la sous-traitance auprès de PME (Foray, 2000b).

Bien qu'en retrait et contestée, la logique des grands programmes, tournés prioritairement vers des ex «champions nationaux », est si prégnante qu'elle se retrouve à l'œuvre dans des 
instruments à visée incitative, tel que le Fonds pour la Recherche et la Technologie (FRT) ${ }^{3}$ : au fil des années, la part des grandes entreprises parmi l'ensemble des crédits distribués par le FRT s'est accrue (40\% en 81, 50\% en 96) (Guillaume, ibid,).

Les difficultés de la coordination de la recherche scientifique publique ou la "mission $\underline{\text { impossible } » d u C N R S}$

A sa création, le CNRS, «le plus grand établissement de recherche du monde », s'est vu confier la mission générale de constituer, de piloter et de valoriser la Science française et ainsi la «faire échapper à la sclérose récurrente des Universités » (Iribarne, 1999, 74). C'est pourquoi le CNRS a vocation à couvrir la totalité des disciplines, «qu'il a été conçu, à la fois comme une agence d'objectifs et une agence de moyens, et qu'il a été mis dans une position carrefour lui demandant d'être capable de constamment rassembler et réagencer des composantes du système français ...»(Iribarne, op.cit.). Pour cela, «il ne peut remplir correctement ses missions qu'en association avec d'autres partenaires » (ibid.).

Or à l'instar des «grands programmes technologiques » la capacité du CNRS à structurer et à réguler le système de recherche est aujourd'hui mise en cause. En particulier, son positionnement vis à vis des organismes de recherche appliquée - INRA, INSERM ... - est en débat dans la mesure où ces derniers ont évolué depuis des activité de «soutien à la profession» vers une recherche de base spécialisée, tandis que les équipes du Cnrs développaient leurs liens contractuels avec les entreprises (Laredo, Mustar, 2001).

En outre, la rigidité des découpages disciplinaires, dans un système national fortement centralisé, rend malaisée l'institutionnalisation de nouvelles disciplines en émergence (Cohen, Le Déaut, 1999, ). Or la capacité à recombiner des savoirs de diverses origines, disciplinaires et institutionnelles, constitue dans le «mode $2 »$ une ressource déterminante pour répondre aux problèmes technologiques des entreprises et, plus largement, de la société. Ainsi la bioinformatique et l'entrepreneurship sont à la fin des années 90 des disciplines reconnues dans des universités américaines tandis qu'elles émergent en France.

Enfin, la prédominance des savoirs académiques tout au long des processus de sélection du système éducatif trouve son prolongement en matière de recherche. C'est ainsi que «les points forts du CNRS correspondent aux disciplines les plus 'nobles' qui sont aussi les plus abstraites, les mathématiques et à un moindre degré, la physique théorique (...). Les points

\footnotetext{
${ }^{3}$ Le FRT est un instrument incitatif dont dispose le MENRT pour développer la partenariat entre la recherche privée et publique.
} 
faibles se trouvent principalement dans des domaines scientifiques à dominante technologique comme ceux qui concernent les sciences pour l'ingénieur ou la biologie appliquée » (Iribarne, 1999), ce qui tend à éloigner des exigences du «mode $2 »^{4}$. Ces traits de la recherche académique ont une résonance dans les représentations qui ont cours dans certains milieux industriels. Ainsi le rapport Guillaume se fait l'écho des doutes des entrepreneurs à l'égard de «l'attitude culturelle des chercheurs à l'égard de la recherche appliquée » (Guillaume, op.cit.).

\section{La dynamique endogène du dispositif public rend celui-ci de moins en moins gouvernable}

Qu'ils soient issus de l'expertise officielle ou de la recherche sur l'innovation - en particulier les travaux de Laredo et Mustar (CSI, 2001) ou de Barré (OST, 2001), les diagnostics avancent que le système français de recherche a fait montre, grâce aux lois de 1982 et de 1984 d'une plasticité indéniable. Celle-ci a certes été bénéfique mais la diversification du système a progressivement fait perdre les avantages de la centralisation en terme de pilotage et de lisibilité. Il en a résulté plus une complexité croissante qu'une capacité réelle de pilotage et d'arbitrage sur les enjeux scientifiques et technologiques.

D'une manière générale, l'action publique, protéiforme et confrontée à la complexité de la réglementation et des dispositifs, a engendré une prolifération d'organismes publics et parapublics dont les missions se recoupent, sinon se recouvrent, au point d'insuffler une vive concurrence inter-institutionnelle là où, selon les attendus initiaux, devaient prédominer coopération et complémentarité des organismes, afin de parvenir à proposer aux destinataires des politiques, entreprises (PME) et chercheurs, un ensemble cohérent de prestations et d'incitations à innover.

Ce déficit de management engendre une très grande difficulté à construire une vision synthétique de l'action publique et, de manière plus restrictive, de l'action de l'Etat. «Cette lacune traduit sans doute le poids pris au sein des administrations centrales par les tâches de gestion au détriment des fonctions de réflexions stratégique et d'évaluation » (Guillaume, op.cit.). De ce fait, le MENRT «n'assure pas un réel suivi de la politique de valorisation des organismes et des établissements [de recherche] »(Guillaume, op.cit).

Dans un paysage institutionnel aussi complexe et enchevêtré, l'évaluation devenue fragmentaire n'aide guère à la formulation d'orientations stratégiques. Or celles-ci sont

\footnotetext{
${ }^{4}$ Il y aurait lieu de pondérer cette explication en se référant à des dimensions historiques et organisationnelles qui tiennent au fait qu'en France, l'engineering ne s'est que très tardivement implantée à l'université, tout en continuant à subir le poids de la coupure entre les écoles d'ingénieurs et le monde universitaire (sur ces questions, voir Grossetti, 2000).
} 
d'autant plus nécessaires que, comme le soulignent Laredo et Mustar (2001b), les Régions et l'Union Européenne, pèsent d'un poids sans cesse croissant en terme de financement ${ }^{5}$. Les difficultés de pilotage de l'action publique régionale en faveur de l'innovation augurent mal de la reconstruction d'une vision d'ensemble. En effet, compte tenu de la multiplicité d'intervenants («beaucoup de conseils généraux et de villes universitaires financent la recherche et la technologie, tant la recherche publique que des actions en faveur de la diffusion technologique »(Guillaume,op.cit.), on aboutit à un double phénomène ; un éparpillement non maîtrisable des interventions publiques et une instabilité des aides apportées aux différents projets, tout ceci pour des montants, au total, importants. La contractualisation (entre l'Etat et les Régions, les grands organismes, les universités ...), en vue de définir des objectifs partagés mobilisant des ressources communes (Gaudin, 1999), ne constitue alors qu'un palliatif très partiel aux déficits de coordination.

Enfin, cette réduction des marges de manœuvre de l'Etat peut se lire dans l'allocation des crédits publics. Ainsi la part des crédits publics destinés à la R/D des entreprises a sensiblement diminué au profit des grands organismes de recherche à tel point que paradoxalement, la part du financement public des dépenses de R\&D des entreprises est désormais plus élevée au Royaume-Uni qu'en France !

$\mathrm{Au}$ total, cette maîtrise, toute relative, du cours de l'action publique tend à justifier les réorientations que nombre d'experts promeuvent pour forger des configurations de « réformateurs ».

\section{LES RECENTES REFORMES : AU DELA DU «MODE 2 » DE PRODUCTION DES SAVOIRS, UN TOURNANT POLITIQUE?}

Un référentiel d'action publique ne peut atteindre l'effectivité souhaitée que s'il est porté et approprié par une configuration d'acteurs pertinente - qui en partagent les lignes de force et les principales traductions opérationnelles - et qui disposent des ressources nécessaires à sa mise en œuvre (Branciard, 2001). De ce point de vue, les réformes engagées en France sont,

\footnotetext{
5 Pour la première fois en 1997, les crédits communautaires ont été d'un montant équivalent aux crédits incitatifs du Ministère de la Recherche. La capacité des acteurs français de la R\&D à obtenir ces fonds communautaires attribués sur projets, par voie d'appels d'offres, est donc essentielle. Or «le taux de retour des fonds européens sur les quatre dernières années n'est que de 15,5\% alors que celui de la Grande-Bretagne est de $20 \%$. La recherche française reste trop hexagonale » (Cohen et Le Déhaut, 1999, 36).
} 
au mieux, au milieu du gué. Pour l'instant, elles concernent essentiellement les relations science-industrie et, plus particulièrement, les créations d'entreprises de haute technologie par des chercheurs publics, ainsi que des mesures destinées à combler les retards français en matière de biotechnologies et de NTICs. Par contre, on en reste encore au stade de la préconisation générale pour réformer les organismes publics de recherche. A ce stade, il ne saurait être question de parler de référentiel, sans une analyse, encore indisponible, des modalités d'appropriation des orientations et préconisations par l'ensemble de la configuration d'acteurs concernée.

Il reste que le processus de production des cadres d'interprétation et d'action peut être retracé. Bien que focalisée, par définition, sur le cas français, la conception de nouvelles orientations politiques en matière de science et de technologie n'exonère pas d'une mise en situation au sein de larges espaces de références, à l'échelle de l'Europe et à celle de l'OCDE. En premier lieu, pour reprendre l'expression récente d'un rapport du Plan (Majoie, 2000), il s'agit de favoriser le bon positionnement de «La France dans la compétition mondiale » grâce au couple «Recherche et innovation ». Là encore, les futures orientations politiques mobilisent des répertoires analytiques fournis par des travaux de recherche mobilisés et réinterprétés par les instances internationales d'évaluation, l'OCDE notamment.

\section{Les nouveaux référents analytiques : de l'interaction au réseau}

Avec l'émergence de modèles théoriques soulignant les interactions entre les différents lieux de production scientifique et technique, dont le "Chain link model" proposé par Kline et Rosenberg (1986) est la figure dominante, la littérature consacrée ces dix dernières années à l'économie de la science et de l'innovation met l'accent sur l'importance des institutions dans l'interaction entre enseignement supérieur, recherche publique et privée, structures de financement et d'intermédiation entre recherche et production de biens ou de services. Ce type d'approche souligne, en outre, les limites de la codification des savoirs et l'importance des 'capacités d'absorption' de chaque protagoniste pour développer des coopérations efficaces en matière de recherche technologique (Lundvall et Johnson, 1994). Elle contribue à justifier la mise en place de consortia de recherche regroupant des partenaires institutionnellement diversifiés. Elle met également en cause la séparation entre recherche fondamentale et recherche appliquée, et l'existence d'une relation causale, dans un schéma linéaire, entre découvertes scientifiques, R\&D industrielle, et marché. Enfin, les critères de partage a priori 
entre recherche publique et recherche privée ont été critiqués par plusieurs auteurs. Notamment, Callon (1994) montre que les investissements publics pour la production scientifique se justifient, non en raison d'une caractéristique intrinsèque de la science comme bien public, mais pour conserver à celle-ci un degré de diversité et de flexibilité permettant à terme de laisser ouvert un plus grand éventail des possibles (Branciard, op.cit.).

Même si la question de la pertinence théorique de ces modèles est récurrente, la force de cette représentation des relations science/innovation tient au fait que, du moins aux Etats-Unis, on observe une configuration d'acteurs scientifiques et industriels immergés dans une multiplicité de réseaux socio-techniques, complexes et hétérogènes ou encore des systèmes localisés qui enchevêtrent des logiques d'action publics et privées, de production de la connaissance et d'innovation technologique. En outre, la fortune croissante et médiatisée d'une forme particulière du Mode 2, illustrée par la Route 128 et la Silicon Valley, fait passer le système de recherche américain, en l'espace d'une décennie, du statut de cas particulier à celui de modèle de référence. Un nouveau concept, celui de «Troisième génération de $R \& D$ » (Mode 3) a théorisé ces pratiques (Roussel, Saad, Eriksson, 1991 ; cité dans Reiger et Wichert-Nick, 1997), pour souligner l'émergence d'une nouvelle organisation de la R\&D non plus « tirée par le marché » mais décentralisée et mise en réseau, cherchant à maintenir une capacité à générer de nouveaux savoirs au-delà du cœur de compétences initiales.

\section{L'interprétation des modèles analytiques en terme d'action publique : «la Triple}

\section{Hélice »}

Les actions politiques devraient alors promouvoir des connexions entre recherche et industrie pour deux motifs : l'un économique, exploiter les capacités scientifiques dans le monde industriel ; l'autre de ressources humaines, un monde académique ouvert et intégré étant plus enclin à produire des professionnalités flexibles et des formations valorisables dans des espaces tant privés que publics.

Ces interdépendances et la diffusion scientifiques et technologiques dans le cadre d'une globalisation de la recherche ont non seulement modifié les techniques constitutives, et parallèlement les formes organisationnelles des différents secteurs mobilisés, mais elles ont suscité la généralisation d'un "modèle" d'interprétation de la production de la science. Il s'agit des travaux dénommés dans la littérature "modèle de la Triple Hélice", avancé notamment par des auteurs américain et hollandais, Etzkowitz et Leydesdorff (1997 et 2000). 
Dans ce modèle, cité par le rapport Guillaume, la "co-production" des connaissances se situerait à l'intersection de trois sphères institutionnelles qui interagissent, l'Université et les organismes de recherche, l'industrie, et les pouvoirs publics. Cette mise en relation des structures de recherche académique avec l'industrie, intégrant les infrastructures publiques de production de savoirs dans les systèmes d'innovation, génèrerait des réseaux trilatéraux par le chevauchement de ces différentes sphères institutionnelles, et l'émergence d'organisations hybrides aux interfaces. L'objectif de ce couplage est de créer un environnement innovateur comprenant des entreprises issues de l'université ou de la recherche (spin off), des initiatives tripartites pour un développement économique fondé sur la connaissance, des alliances stratégiques entre des firmes de différentes tailles et à différents niveaux de technologie, des laboratoires publics, et des équipes de recherche universitaires. En favorisant la fondation de pôles scientifiques et industriels au niveau local qui transcendent les frontières traditionnelles institutionnelles (public/privé, académique/appliqué etc...), ces interventions publiques s'inscriraient dans une logique d'accumulation organisée des connaissances et de création de capacités d'innovation au niveau macro-économique (Branciard,op.cit.)).

La dynamique de ce modèle implique des transformations internes dans chacune des trois sphères tout autant que l'intensification de leurs interrelations. L'un des obstacles soulignés à son application est notamment le besoin croissant, pour le changement technologique, de nouvelles disciplines ou de recombinaisons de disciplines créant des champs de connaissances pertinents, ce qui peut signifier une remise en cause de l'organisation du secteur de production de la science (Universités et Organismes de recherche) et de l'identité de ses acteurs.

$\mathrm{Au}$ bout du compte, ces constructions analytiques et leurs reformulations en terme de politique publique semblent illustrer le développement d'une organisation en réseau, fondée sur les initiatives des acteurs autour de projets, que Luc Boltanski et Eve Chiappello (1999) identifient comme emblématique des principes qui animent les transformations du capitalisme contemporain. Il est symptomatique que les compétences individuelles des «nouveaux travailleurs de la recherche » inscrits dans le «mode $3 »$ de production des savoirs se formulent en terme de « compétences pour coopérer, pour travailler en réseau, pour combiner des savoirs différenciés » (Lam, 2001). Il y aurait donc émergence, dans le champ privilégié de l'innovation et de la recherche, d'une nouvelle philosophie politique de la conduite des activités scientifiques et économiques. En outre, l'ensemble de la littérature insiste sur la confiance, l'importance des relations personnelles pour soutenir le fonctionnement du réseau. 


\section{Les traductions « expertes » en vue de l'élaboration d'un référentiel d'action publique}

Ces cadres analytiques et interprétatifs inspirent certains des principes politiques qui président à la mise en mouvement du système français de recherche et d'innovation. Il sont intégrés dans une approche modernisatrice plus « classique » pour la réforme «à la française », présentant des référents qui entremêlent le «vieux » et le neuf ».

Une première orientation, fort classique, s'inscrit dans la longue lignée de la nécessité d'une modernisation des institutions et des organisations (Jobert et Muller, 1987) destinée à adapter telle ou telle composante du système économique national «aux évolutions que connaissent tous les pays industrialisés, en raison [en l'occurrence] de l'évolution rapide des marchés et des techniques, de la pression des contraintes budgétaires, de l'externalisation croissante de leur recherche-développement par les grandes entreprises » (Guillaume,op.cit.). Une norme de compétitivité hors coûts constitue ainsi le référentiel global des transformations qu'une nouvelle action publique doit promouvoir, sous peine d'exposer les acteurs économiques nationaux à un « retard » important vis à vis de leurs concurrents.

Pour ce qui est de la recherche, ce diagnostic renoue ainsi avec les années trente qui s'étaient achevées sur la création du CNRS, à la suite de constats sévères de grands scientifiques, d'après lesquels «le retard pris par la recherche française par rapport à ses concurrentes directes, notamment l'Allemagne était considérable »(Cohen, Le Déaut, 1999).

La seconde orientation est sans doute plus originale dans le contexte français puisqu'elle conduit le rapporteur à faire part de sa «conviction générale que la capacité d'entreprendre, d'innover, de diffuser les technologies nouvelles, plus qu'à d'autres moments de l'histoire économique, constitue un déterminant essentiel de la compétitivité, de la croissance et de l'emploi» (ibid.). Le recours à la «conviction» dénote bien d'un changement partiel de référent accordant aux liens de la science avec les entreprises et à la mise en place de réseaux coopératifs une préférence sur une plus classique rhétorique civique du bien fondé d'un investissement dans une Science indépendante du politique et du monde économique.

Sur la base des travaux de David et Foray (1995), cette approche relève le fait que les politiques de la science et de la technologie occidentales ont plutôt mis l'accent sur la stimulation de la production de nouveaux savoirs que sur leur diffusion. La mise en place de mécanismes institutionnels soutenant une diffusion plus large et un accès non coûteux aux nouveaux savoirs est alors considérée comme cruciale parmi les déterminants de la capacité organisationnelle d'un dispositif public. En conséquence, les pouvoirs publics doivent 
développer un rôle de régulateur et plus encore de coordonnateur « en réduisant les obstacles qui empêchent la formation de réseaux et en veillant à ce que l'infrastructure de la recherche publique fonctionne en étroite collaboration avec le secteur des entreprises » (OCDE revue «Synthèses », juin 1999) «Promouvoir le progrès scientifique et technologique »).

On est ainsi de plain pied dans le monde de la «Triple Helice » d'Etzkowitz et Leydesdorff (1997 et 2000) décrit plus haut. Plus précisément, il s'agit de passer d'un mode de fonctionnement de «l'hélice», reposant sur le modèle du « grand programme technologique » - modèle de la Triple Hélice I de l'Etat entrepreneur -, qui regroupe une agence publique, une institution de recherche et un grand groupe industriel, à un fonctionnement en réseau interactif où les acteurs réalisent des apprentissages organisationnels fondés sur la coopération - soit le modèle de la Triple Hélice III de l’Etat animateur - (voir Branciard, 2001a) .

\section{L'étalonnage international : la référence américaine réinterprétée par l'OCDE}

$\mathrm{Au}$ regard de l'étalonnage des recommandations de l'OCDE (2000), tous les registres de l'action publique sont appelés à se réformer dans le cas de la France.,

La démarche d'ensemble de l'OCDE, appuyée sur les «bonnes pratiques » qualifiées comme telles au regard de leurs performances en matière de compétitivité, constituent un «mix » particulièrement complexe entre plusieurs inspirations théoriques dont la compatibilité ne va pas complètement de soi : 1. Une approche par les incitations et, en particulier, par les droits de propriété qui vise à pallier les défaillances de l'organisation privée comme de l'intervention publique, en favorisant des comportements conciliant l' "open science » et la reconnaissance des performances individuelles 2 . Une conception des liens entre recherche fondamentale, recherche appliquée et innovation, qui s'appuie sur des interactions et coopérations entre l'Académie et l'Entreprise, en rupture avec le classique modèle linéaire (Mode 2 et Chain link model de Kline et Rosenberg) 3. Une articulation des pouvoirs publics, de la recherche et de l'industrie ( Triple Hélice III ) qui favorise l'émergence de milieux innovateurs, en particulier sur une base localisée (émergence de réseaux ).

Cette référence explicite à la trajectoire à succès américaine n'est pas dénuée d'ambiguïté puisque cette «success story » est intimement liée à l'échelle dans laquelle elle a été produite, mais en outre elle a fortement bénéficié d'un contexte macro-économique et boursier préalable très favorable à des anticipations positives et à des prises de risque des acteurs qui soient de nature à faciliter les convergences des intérêts publics et privés . 


\section{Les réformes et les premières étapes de la mise en œuvre : combler le retard, grâce à la mobilisation des acteurs au sein de nouveaux réseaux}

La loi sur la recherche et l'innovation de juillet 1999 est d'une essence très différente de celle de 1982. Bien que les exposés des motifs ministériels tout comme les rapports parlementaires introductifs aux débats législatifs prennent le soin d'inscrire les nouvelles dispositions dans la filiation des lois de 1982 et de 1984, elle vise à ouvrir des espaces favorables à la réalisation de projets technologiques en promouvant la création d'entreprises de haute technologie par les chercheurs, et en suscitant la création de réseaux mariant acteurs publics et privés. Le domaine des biotechnologies étant, de ce point de vue, exemplaire. Cette rhétorique de la continuité a pour but de conforter une justification civique de réformes très directement inspirées de l'exemple américain en matière de start up et de spin off, relayé par l'OCDE. Il s'agit d'une sorte de «traduction », dans le contexte français, de référents qui visent à rendre plus poreuses les frontières entre monde marchand et non marchand. L'intention est de construire un compromis légitime entre une «mutation» vers une plus grande efficacité économique et une inscription dans les conceptions politiques françaises prédominantes sur l'indépendance de la science vis à vis du monde des affaires. C'est la raison pour laquelle le terme de «tournant» nous paraît assez adapté à une évolution en forme de « rupture douce et progressive » avec le cours antérieur, une sorte de bifurcation au sens «évolutionniste » du terme. D'où la volonté d'encadrer les opportunités de profit, concrétisée par le refus de l'Assemblée Nationale d'accorder des réductions d'impôt, votées par le Sénat, aux investisseurs dans le capital de sociétés innovantes, et les dispositions sur les stocks options.

\section{Promouvoir la création d'entreprises de haute technologie et favoriser l'entrepreneuriat} universitaire

Quatre volets structurent cette orientation. Le premier concerne les collaborations entre personnels de recherche et entreprises. Il permet à un chercheur ou à un enseignantchercheur de créer une entreprise, de participer à son capital, de la diriger ou de lui apporter son concours scientifique, tout en conservant son statut de fonctionnaire et en pouvant continuer à entretenir des liens avec son laboratoire d'origine.

Le second est consacré aux relations entre organismes de recherche ou universités et entreprises. Il prévoit la création de services d'activités industrielles et commerciales par les OPR et les universités, et celle d'incubateurs et de fonds d'amorçage. Ces incubateurs (31 labellisés en 2001) pourront mettre à disposition de jeunes entreprises technologiques, 
moyennant rémunération et pour une période de temps limitée, des locaux, des équipements et des matériels (Bret, 1999) ${ }^{6}$. En outre, la volonté de «développer une culture du risque » (Bret,op.cit.) s'est concrétisée par le lancement de fonds d'amorçage (avances en capital) dont le but est d'accompagner les créateurs d'entreprises dans la phase d' «incubation » de leur société. Trois fonds nationaux thématiques et sept fonds régionaux d'amorçage ont été sélectionnés dans le cadre de l'appel à projets 1999. En outre, deux fonds hors appel à projets ont été créés par l'INRIA et le CEA. Le troisième volet concerne l'instauration d'un cadre fiscal favorable aux entreprises innovantes, le quatrième la constitution d'un cadre juridique adapté par l'ouverture du régime des Sociétés par Actions Simplifiées SAS.

\section{Instrumenter la connexion entre la recherche publique et les entreprises}

Il ne s'agit plus de mettre sur pied de grands programmes mais d'inciter à la constitution de projets précis de collaboration firmes-recherche académique dans des domaines jugés prioritaires, les NTICs et les biotechnologies. Cette optique se traduit par un changement sémantique et institutionnel : on se réfère au «réseau » plutôt qu'au «programme »; on s'appuie avant tout sur les Actions Concertées Incitatives et l'appel à projets. La formule n'est pas très éloignée des consortia de recherche industrielle «sans les murs », prônés par le rapport Guillaume et associant des grandes entreprises, des PMI, des laboratoires de recherche et des opérateurs du transfert technologique, sur des thèmes répondant à un besoin fonctionnel, défini à l'initiative de l'ensemble des industriels et indispensable à leur capacité d'innovation (Guillaume, 1998) ${ }^{7}$.

Cette formule institutionnelle répond à l'une des recommandations de l'OCDE : «il conviendrait d'éviter de compenser les distorsions existantes (manque de cohérence entre administrations, accès insuffisant des PME aux programmes publics) en y superposant de nouvelles (création d'organismes de coordination des organismes existants, mesures supplémentaires pour les PME sans toucher aux sources de biais en faveur des grandes entreprises) (OCDE, 1999).

\section{Les incertitudes sur le cours à venir de l'action publique : entre transposition et accommodation sociétales}

\footnotetext{
${ }^{6}$ Les « incubateurs doivent être, pour de jeunes créateurs, des lieux d'information et de mobilisation d'expertises, de conseils juridiques, commerciaux, en management, de moyens technologiques et de financements » (Chevallier, 1999, 17), ainsi que de soutien logistique et d'accompagnement des créateurs dans l'élaboration de leurs projets, et en amont des instances de détection et d'évaluation des projets de création d'entreprise.

${ }^{7}$ Le référent en la matière est constitué du réseau des Fraunhoffer allemandes.
} 
$\mathrm{Au}$ total, dans cette phase que l'on peut qualifier de préliminaire, la France semble être « une bonne élève », au regard des nouveaux standards internationaux, dans la mesure où elle a engagé nombre de transformations conformes aux recommandations de l'OCDE (OCDE, 2000). Il reste que le sens et le cours de cette nouvelle action publique en matière de «science, de technologie et d'innovation » sont loin d'être stabilisés (Branciard, 1999). En effet, des différents points de vue évoqués ci-dessus, le «tournant à la française » comporte des ambiguïtés :

- L'approche linéaire des relations Science-Industrie est d'autant moins absente que continue à s'exprimer une approche de la recherche publique qui conçoit celle-ci comme étant le support d'une sorte de «gisement» de découvertes qu'il convient de «valoriser » : l'Etat en étant le dépositaire et donc, peu ou prou, le propriétaire, il lui revient d'être le prescripteur de la valorisation efficace de ce bien de la Nation

- Le point de vue symétrique, qui met l'accent sur le pilotage par l'aval, méconnaît la complexité des interactions entre recherche fondamentale et recherche privée et peut favoriser les injonctions plus ou moins autoritaires à travailler avec (pour) les entreprises, au risque de déstabiliser les assises de la recherche de base

- L'approche mobilisatrice, qui vise à combler, au plus vite, le retard pris sur les compétiteurs étrangers et désigne le niveau national comme le lieu privilégié de la cohérence, est assortie d'un double risque : restaurer subrepticement la logique du « grand programme » d'une part, promouvoir une logique d'aménagement du territoire au sein des réseaux et autres dispositifs incitatifs publics, potentiellement contradictoire avec une démarche privilégiant les initiatives «bottom up », porteuses de processus d'innovation par foisonnement.

En fin de compte, ces interrogations peuvent être synthétisées par une question sur la nature du «benchmarking societal» qui est et sera développé, dans le cours de l'action publique. Pour reprendre Lundvall et Borras (1997), s'agit-il de faire des emprunts directs («institutional borrowing») aux Etats-Unis, une sorte de transposition par décret, ou de réaliser des apprentissages ( institutional learning ») favorisant les appropriations locales ? Dans le second cas, l'ensemble des mesures mises en œuvre relèverait, en quelque sorte, d'un «benchmarking contextualisé » ( Lundvall, 2001). Il revient à puiser dans un répertoire de mesures fourni par les expériences étrangères, en les adaptant pour les rendre compatibles 
avec le contexte sociétal dans lequel elles sont appelées à prendre sens. Au bout du compte, compte tenu des héritages sociétaux («path dependency») et du caractère plus ou moins autoritaire et adapté des nouvelles réglementations (qui par exemple, variera fortement d'un secteur à l'autre), trois scénarios de référence peuvent être identifiés pour interpréter le cours des réformes institutionnelles. Soit, en s'inspirant de Casper (1999), des processus :

- de convergence vers un modèle de type américain, ce qui supposerait, pour un pays comme la France, une transformation structurelle des règles, institutions et conventions

- de spécialisation qui signifierait un renforcement du modèle et de la position sociétale au sein de l'espace mondial de la science, de la recherche et de l'innovation

- d'accommodation du cadre actuel afin d'implanter certaines formes d'entrepreneuriat scientifique, tout en préservant certaines capacités sociétales existantes.

Ce troisième scénario est sans doute celui qui se rapproche le plus du cours actuel. La problématique du retard à combler rend assez peu probable un rupture franche avec la configuration actuelle, ce qui consisterait à abandonner totalement les avantages de la «mission-oriented policy ». Comme le soulignent Amable, Barré et Boyer (1997), le système fondé sur les grands programmes - composante pour ces auteurs d'un modèle d'intégration européenne appuyé sur l'intervention publique - «trouve sa logique dans les périodes où un pays en retard cherche à se doter des institutions nécessaires à combler son handicap technologique ». En ce domaine, la voie est très étroite pour l'action publique à la française qui requiert à la fois une mobilisation destinée à combler un retard et l'introduction de nouveaux repères cognitifs et institutionnels.

\section{Conclusion}

Par la médiation des rapports d'experts, en particulier de l'OCDE, les analyses savantes de la science, de la technologie et de l'innovation, concourent à forger des diagnostics très critiques sur l'état et l'avenir du dispositif français de recherche et à en tirer des préconisations pour l'action. Au stade des intentions tout du moins, il en résulte une inflexion significative de l'action publique en faveur d'une nouvelle créativité technologique. Elle repose sur de nouvelles incitations des chercheurs à valoriser technologiquement et industriellement leurs recherches. Elle s'appuie également sur des dispositifs incitant entreprises et équipes de 
recherche publique à constituer des réseaux, appelées à engendrer tant des connaissances que des start up.

Mais l'inertie organisationnelle se manifeste dans les difficultés, pour la puissance publique, à passer d'une posture d'un «Etat entrepreneur» à celle d' un «Etat animateur» (voir le cas de la génomique), favorisant un développement technologique par «foisonnement» (Branciard, 1999, 2001). Un basculement brutal vers une construction «à l'américaine » semble peu viable. Par contre, une accommodation au contexte français des expériences extérieures réussies semble plus réaliste : toute la question est alors de savoir si elle est susceptible de se réaliser à un rythme compatible avec l'ampleur du retard à combler, problématique qui sous-tend cette volonté de réforme. Il serait donc imprudent d'assimiler les changements en cours comme annonciateurs de la victoire inéluctable de la référence marchande. Si l'on se réfère à «la cité par projets » de Boltanski et Chiapello (1999), c'est plutôt un nouveau principe «connexionniste » de formulation et d'organisation de l'action publique, appuyé sur des réseaux d'acteurs publics et privés qui tend à s'imposer. La composition de ce principe avec la régulation marchande prendra manifestement des formes variables de part et d'autre de l'Atlantique; aux Etats-Unis, plus articulée autour d'une régulation fondée sur l'identification précise des droits de propriété ; en Europe, plus liée à une coopération entre les acteurs, en vue de produire des apprentissages organisationnels et institutionnels.

La réorganisation interne du dispositif de recherche publique n'en est qu'à ses prolégomènes Il est encore trop tôt pour apprécier les premiers impacts du nouveau cours, censé être impulsé par les récentes réformes, sur sa cohérence d'ensemble. Enfin, curieusement compte tenu de la référence constante à la mondialisation, l'insertion de la politique nationale dans une approche européenne est restée très peu présente dans les débats parlementaires : elle semble pourtant incontournable dans le cas de la génomique (Branciard 2001b), au-delà du registre de son seul rendement budgétaire - faire en sorte que la recherche française bénéficie de crédits européens supérieurs au montant de la contribution de l'Etat français ! -.

Plus généralement, cette construction cognitive des politiques publiques révèle un processus contradictoire. D’un côté, la convergence et la récurrence des diagnostics critiques constituent progressivement un ensemble de croyances ou d'images qui engendre de l'irréversibilité, au sens où le statu quo semble devenir intolérable. De l'autre, aussi standardisée soit-elle par les soins de l'OCDE, cette production savante donne lieu à des ré-interprétations multiples qui ouvrent la voie à des bifurcations singulières des trajectoires sociétales, au prix d'apprentissages et d'accommodements complexes. Aller plus loin demanderait que l'on 
retrace beaucoup plus finement les configurations d'experts qui ont porté ce nouveau cours dans les différents pays.

\section{Bibliographie}

AMABLE B., BARRE R., BOYER R., 1997, Les systèmes d'innovation à l'ère de la globalisation, Paris, Economica

ATTALI J., 1998, Pour un modèle européen d'enseignement supérieur, rapport au Ministre de l'éducation nationale, de la recherche et de la technologie, miméo.

BARRE R. et PAPON P., 1998, La compétitivité technologique de la France, in Guillaume, Henri, 1998, Rapport de mission sur la technologie et l'innovation, remis au Ministre de l'éducation nationale, de la recherche et de la technologie, au Ministre de l'Economie, des Finances et de l'Industrie et au Secrétaire d'Etat à l'Industrie, miméo, Paris, 216-227.

BARRE R., THERY J-F., 2001, La loi sur la recherche de 1982 : origines, bilan et perspectives $d u$ "modèle français », coll. Sciences en questions, INRA Editions, Paris.

BRANCIARD A., 1999, L'articulation science-innovation en France: dix ans d'action publique pour le développement de la génomique et des biotechnologies, miméo LEST nov.1999, publié dans Les Cahiers de l'innovation, n05/2000, CNRS, 37 p..

BRANCIARD A., 2001a, Politiques publiques et espace d'innovation dans la biologie, étude de dispositifs d'intégration science/industrie et de création d'entreprises: le cas de la génopole d'Evry, miméo LEST, Aix en Provence, Rapport de recherche pour le Programme CNRS «Enjeux économiques de l'innovation », 121 p..

BRANCIARD A., 2001b, Les avatars de la génomique française, Biofutur $\mathrm{n}^{\circ} 216$, novembre, pp. 32-34.

BRET J-P., 1999, Rapport sur la loi sur l'innovation et la recherche, Commission des affaires culturelles, familiales et sociales, Assemblée Nationale, Paris.

CASPER S., 1999, National Institutional Frameworks and High-Technology Innovation in Germany. The Case of Biotechnology. Berlin : Working Paper Wissenschaftszentrum Berlin für Sozialforschung.

CHEVALLIER D., 1999, Introduction au débat de la commission de la production et des échanges de l'Assemblée Nationale en vue d'un avis sur le projet de loi sur l'innovation et la recherche, Assemblée Nationale, n 1619, 26 mai 1999. 
COHEN P., Le DEAUT J. Y., 1999, Priorités à la recherche, 60 propositions pour la synergie entre recherche et enseignement supérieur, la mobilité et les échanges, l'évaluation et l'autonomie des jeunes, Rapport de mission au Premier Ministre, miméo.

COMMISSION DES COMMUNAUTES EUROPEENNES, 1993, Croissance, compétitivité, emploi : le défis et les pistes pour entrer dans le XXIe siècle, Livre blanc

COUR DES COMPTES, 1997, Les activités de valorisation des établissements publics à caractère scientifique et technique, Coll. Rapports Publics, Journaux Officiels, Paris.

ERGAS H, 1992, A future for Mission-oriented Industrial Policies ? A Critical review of Developments in Europe, Paris, OECD.

ETZKOWITZ H., LEYDESDORFF L., 1997, Universities and the Global Knowledge Economy. A triple Helix of University-Industry-Government Relations, Pinter, London and Washington.

ETZKOWITZ H., LEYDESDORFF L., 2000, The dynamics of innovation: from National Systems and 'Mode 2' to a triple helix of university-industry-government relations, Research Policy 29, pp.109-123.

FORAY D., 2000, Inerties institutionnelles et performances technologiques dans la dynamique des systèmes d'innovation : l'exemple français in Tallard M ., Théret B., Uri D, Innovations institutionnelles et territoires, coll. Logiques Politiques, L'Harmattan, Paris, 81100.

FORAY D., 2000b, L'économie de la connaissance, Coll. Repères, La Découverte, Paris.

GAUDIN J. P., 1999, Gouverner par contrat, l'action publique en question, Presses de Sciences Po, Paris.

GIBBONS M. (ed.), 1994, The new Production of Knowledge, The dynamics of Science an research in contemporary societies, Sage, Londres.

GROSSETTI M. (2000), La construction d'espaces universitaires hybrides : les sciences pour l'ingénieur à travers les exemples de l'informatique et du génie chimique, XVI congrès de l'AISLF, 3-7 Juin, Québec.

GUILLAUME H., 1998, Rapport de mission sur la technologie et l'innovation, remis au Ministre de l'éducation nationale, de la recherche et de la technologie, au Ministre de l'Economie, des Finances et de l'Industrie et au Secrétaire d'Etat à l'Industrie, miméo, Paris. IRIBARNE (d') A., Le CNRS. Propositions d'analyse pour une réforme. Annales des Mines : gérer et comprendre, septembre 1999, $\mathrm{n}^{\circ} 57, \mathrm{p} .58-80$.

JOBERT B. et MULLER P., 1987, L'Etat en Action, PUF, Paris. 
KLINE S.J., ROSENBERG N. (1986), An overview of innovation, in The positive sum strategy, ed. by Landay R and Rosenberg N, Academy of Engineering Press.

LANCIANO C., 2001, Les logiques de relation entre les acteurs de l'innovation: Entreprises-Systèmes d'enseignement supérieur et de recherche-Action publique, Draft Paper, SESI Project, Lest, Aix en Provence.

LAREDO P., MUSTAR P., 2001a, La nouvelle donne des politiques publiques, BIOFUTUR $\mathrm{n}^{\circ} 216$, novembre, pp. 22-26.

LARÉDO P., MUSTAR P., 2001b, French research and innovation policy : two decades of transformation, in Research and Innovation Policies in the New Global Economy: An International Comparative Analysis, ed by Laredo P and Mustar P, Edward Elgar Publishing LUNDVALL B. A., BORRAS S., 1997, The globalising learning economy: Implications for innovation policy, DG XII, EC, Brussels.

LUNDVALL B. A., 2001, Benchmarking and policy learning, Key-note address for the Conference The contribution of socio-economic research to the benchmarking of RDT policies in Europe, EC, Brussels, March 15-16.

MAJOIE B. (coord.), 2000, Recherche et innovation: la France dans la compétition mondiale, Rapport du groupe de travail au Commissaire au Plan, La Documentation Française.

MUSTAR P., LARÉDO P., 2001, Innovation and research policy in France (1980-2000) or the disappearance of the Colbertist state, Research Policy, 31, 55-72.

NOHARA H., 2001, Joint Production of Competences between Academia and Industry as a Bridging Institution in emergence, in Verdier Eric (coord.) Higher Education Systems and Industrial Innovation, SESI Project, TSER Programme, Lest, Aix en Provence.

OCDE, 1999, France, Etudes économiques, (voir Chapitre III «La politique de recherche et d'innovation »), Paris, OCDE.

OCDE, 2000, Perspectives de la Science, de la Technologie et de l'Industrie (OECD Science, Technology and IndustryOutlook 2000), Paris.

OST, 2000, Science et Technologie, indicateurs 2000, rapport de l'Observatoire des Sciences et Techniques, sous la direction de Barré, Rémi, Economica, Paris.

PAPON P., 1998, Research Institutions in France :Between the Republic of Science and the Nation-state crisis, Research Policy, 27, 771-780.

PESTRE D., 1997, La production des savoirs entre économie et marché, Revue d'Economie Industrielle, $\mathrm{N}^{\circ} 79$ 
SHINN T., 2002, Nouvelle production du savoir et triple hélice : tendances du prêt-à-penser les sciences, Actes de la Recherche en Sciences Sociales, $\mathrm{N}^{\circ}$ spécial Science.

TREGOUET J., Introduction au débat de la commission des finances du Sénat en vue d'un avis sur le projet de loi sur l'innovation et la recherche, Sénat.

VAN DYCK L., 2001, Quelle recherche pour l'Europe ?, Biofutur n 216, pp. 35-38. 